\title{
Observation and Modelling of Barrel Droplets on Vertical Fibres Subjected to Gravitational and Drag Forces.
}

\author{
Benjamin J. Mullins ${ }^{1,2} *$, Roger D. Braddock ${ }^{2}$, Igor E. Agranovski ${ }^{2}$, Roger A. Cropp ${ }^{2}$ \\ and Gerhard Kasper
}

1. Universitt Karlsruhe (TH), Institut für Mechanische Verfahrenstechnik und Mechanik (MVM), Gas-Partikel-Systeme, 76128 Karlsruhe, Germany.

2. Faculty of Environmental Sciences, Griffith University, Nathan QLD 4111 Australia.

Date: $X^{\text {th }}$ October 2005

* corresponding author.

Benjamin Mullins

Universität Karlsruhe (TH)

Institut für Mechanische Verfahrenstechnik und Mechanik (MVM)

Gas-Partikel-Systeme

D-76128 Karlsruhe

Germany

Phone: $+49(0) 7216086567$

Fax: +49(0) 7216086563

Email: benjamin.mullins@mvm.uni-karlsruhe.de 


\section{Abstract}

Extensive experimental investigation of the wetting processes of fibre/liquid systems during air filtration (when drag and gravitational forces are acting) has shown many important features, including droplet extension, oscillatory motion, and detachment or flow of drops from fibres as airflow velocity increases. A detailed experimental study of the aforementioned processes was conducted using glass filter fibres and $\mathrm{H}_{2} \mathrm{O}$ aerosol, which coalesce on the fibre to form barrel droplets. The droplets were predominantly observed in the Reynolds transition (or unsteady laminar) flow region,. The droplet oscillation appears to be induced by the onset of vortexes in the flow field around the droplet as the increasing droplet size increases the Reynolds number. flow in this region is usually modelled using the classical Two dimensional Karman vortex street, and there exist no 3D equivalents. Therefore to model such oscillation it was necessary to create a new conceptual model to account for the forces both inducing and inhibitingsuch oscillation. The agreement between the model and experimental results is very good for both the radial and transverse oscillations.

Keywords: Fibre Wetting, Drag, Filtration, Liquid Aerosols 


\section{Introduction}

Fibre wetting processes are important in a diverse range of industries, including, composite manufacture, textile manufacture and cleaning, wire coating, and other areas. However in such cases there are rarely any forces, other than interfacial forces and gravity, acting on the fibre droplet system. In [naughty boy - never, never, never start a sentence with 'and' (or is this the German influence??) much of the theory even gravitational forces are neglected, with only the interfacial three phase forces (solid/liquid liquid/gas interfacial tension) being considered. ????? The work of Kumar and Hartland $[1,2]$ is one of the few studies which have identified the effect of gravity on large droplets on fibres. However during filtration of liquid aerosols, both airflow and drag forces must be considered. A discussion of wet filtration and the filtration of liquid aerosols is given in previous literature [3, 4].

The spectrum of possible droplet shapes which occur during fibre wetting has been previously detailed in the literature [5], with barrel and clamshell droplets being the two principal types that occur. Clamshell droplets are categorised by their axial asymmetry and a contact angle of $\geq 90^{\circ}$. Barrel shaped droplets - possessing a higher interfacial energy than clamshell droplets - have a lesser contact angle and are regarded as axisymmetric (on a vertical fibre or when gravitational forces are neglected). It will be shown later in this work that barrel droplets become axially asymmetric when drag forces are acting on them.

In the previous microscopic scale work $[3,4,6]$, many important features were observed, relating to droplet formation (coalescence from aerosols), motion and flow along the fibre. One of the key features observed, which is vital to quantifying the flow field around filter fibres, is the oscillation of droplets as Reynolds number increases. This paper details the 
development of a model for the motion of barrel droplets on fibres in the Reynolds transition flow region. The accurate study of droplet motion in this flow region is important since in a fibrous filter system most droplets will remain present on the fibres until the flow around them reaches this region. The motion of such droplets can be expected to have a significant effect on the flow and particle capture inside a filter.

This paper will experimentally examine the unique and interesting features related to the motion of barrel droplets on filter fibres, and compare experimentally imaged oscillations with the model developed. As stated previously, barrel droplets are defined by a contact angle less than $90^{\circ}$ and usually have an infinitesimally thin film connecting the droplets. This work is an extension of previous work which examined the oscillation of clamshell droplets [6], and the same model will be adapted for this work. 


\section{Model Development}

Figure 1 shows the components of the droplet oscillation. This oscillation is believed to be induced by a change in the flow field around the droplet from the laminar to the transition or unsteady laminar regime. This is supported by the Reynolds number at which such oscillations occur. Flow in this region is usually modelled by the use of the two-dimensional Karman vortex street,. However, there is no 3D equivalent analytical flow field known, and another method must be devised (it should be noted that some commercial CFD packages can resolve vortexes flow fields numerically in 3D). A model for the droplet oscillation has thus been developed [6] by considering the forces acting on the droplet, and taking the tension force at the line of contact between droplet and fibre $(T)$ as a restoring force counteracting drag (Figure 2).

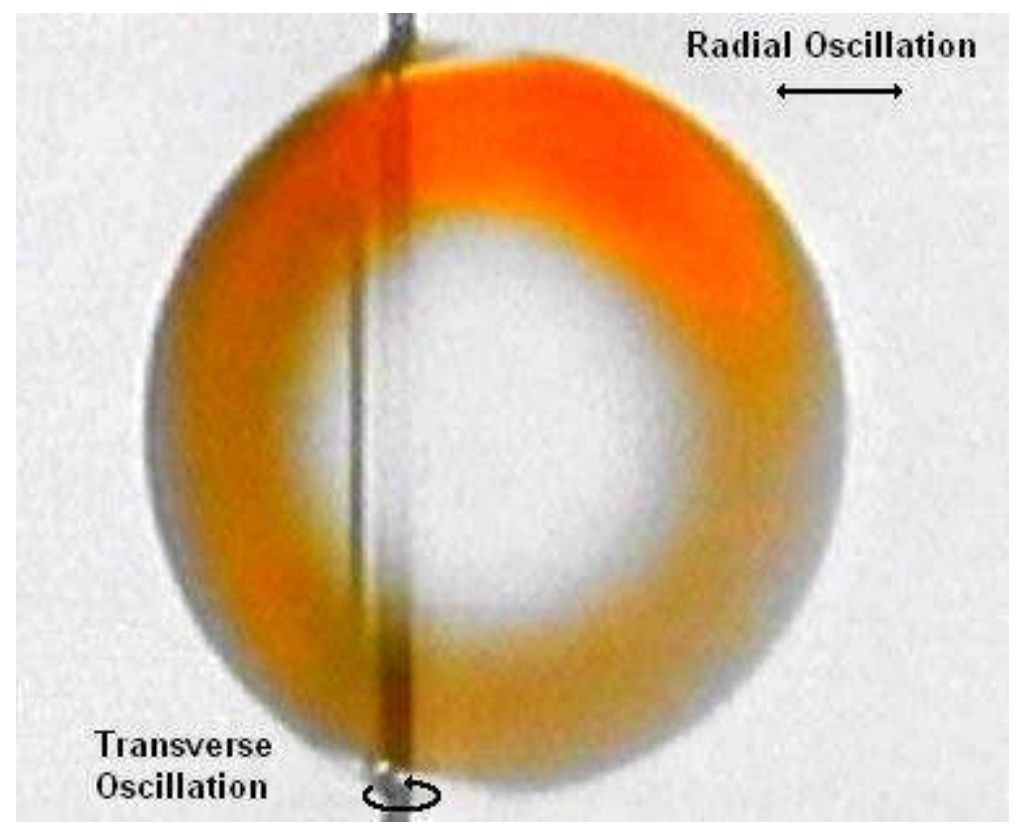

Figure 1. Barrel droplet on $7 \mu \mathrm{m}$ glassfibre fibre showing components of droplet oscillation. It will be noted that the droplet is no longer axisymmetric due to drag forces. When airflow velocity $=0$ the droplet returns to the axisymmetric position. 


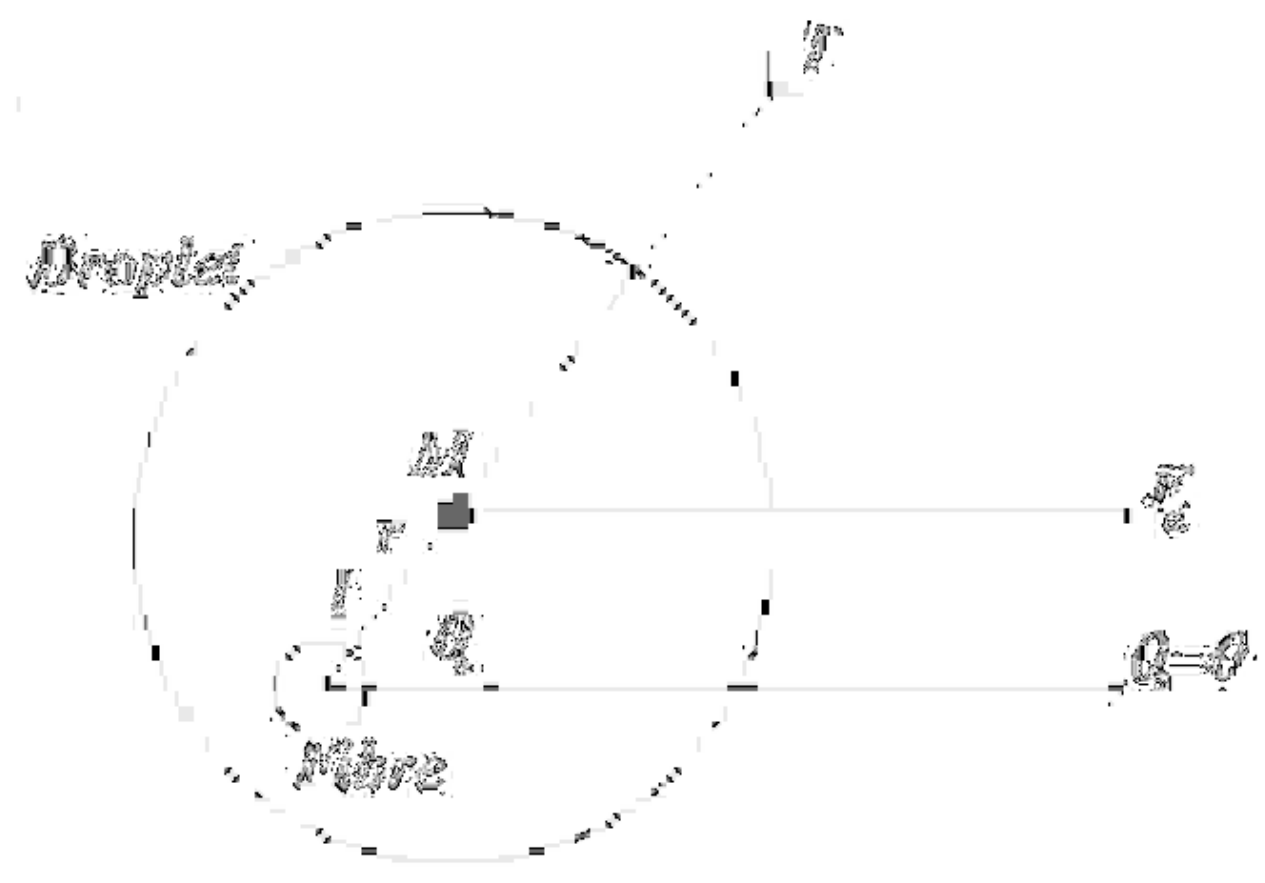

Figure 2. Diagram of droplet oscillation in a plane perpendicular to fibre, showing forces acting on droplet. This is repeated in the text

Figure 2 gives a graphical representation of the terms used in the model, where $M$ is the droplet mass centre, $r$ is the displacement of $M$ from the at rest position, $l$ is the displacement of the rest position from $M, T$ is the tension (restoring force), $F_{d}$ is the air drag force on the droplet, and $\theta_{o}$ is the angle of the droplet relative to the airflow direction. The equations governing the droplet oscillation, can be written in radial and transverse co-ordinates [7],, as

$m\left(\ddot{r}-r \dot{\theta}_{o}^{2}\right)=-T+F_{d} \cos \theta_{o}+\alpha_{o} \cos \left(\omega_{o} t+\phi\right)$,

and

$m\left(r \ddot{\theta}_{o}+2 \dot{r} \dot{\theta}_{o}\right)=-F_{d} \sin \theta_{o}+\beta_{o} \sin \left(\omega_{o} t+\phi\right)$,

where the dot above variables denotes derivation with respect to time, and 
$T=\left\{\begin{array}{cc}0 & r \leq 0 \\ \lambda(r) & r \geq 0\end{array}\right\}$

and the oscillation forcing terms $\alpha_{o} \cos \left(\omega_{o} t+\phi\right)$, and $\beta_{o} \sin \left(\omega_{o} t+\phi\right)$ represent the drag effects of the transition zone air stream. The model assumes the droplet is essentially rigid. The droplet rigidity with respect to droplet rotation has been discussed in previous work [3]. Although large droplets (which oscillate) usually possess lower Ekman numbers (E) than smaller droplets, the values ([3]) are still high enough that the rigid body assumption is valid.

Observations (Figure 1) show how the droplet is displaced downstream of its axisymmetric position on the fibre by airflow. At rest the droplet will be axisymmetric $(l=0)$. Initiating air flow displaces the droplet further downstream (ie increases $r$ ). The restoring tension, $\mathrm{T}$ which uses the spring-like restoring constant $\lambda$ is assumed to be linear. These equations (1-3) can then be transformed to vector form,

$\frac{d u}{d t}\left[\begin{array}{l}u_{1} \\ u_{2} \\ u_{3} \\ u_{4}\end{array}\right]=\left[\begin{array}{c}u_{2} \\ u_{1} u_{4}^{2}-\frac{\lambda}{m}\left(\begin{array}{c}u_{1}-l \\ 0\end{array}\right)+\frac{F_{d}}{m} \cos \left(u_{3}\right)+\frac{\alpha_{o}}{m} \cos \left(\varpi_{o} t+\phi\right) \\ u_{4} \\ \frac{-1}{u_{1}}\left(\left(2 u_{2} u_{4}+F_{d} \sin \left(u_{3}\right)\right) / m\right)+\frac{\beta_{o}}{m u_{1}} \sin \left(\varpi_{o} t+\phi\right)\end{array}\right]$,

using $u_{1}=r, u_{2}=\dot{r}, u_{3}=\theta_{o}$, and $u_{4}=\dot{\theta}_{o}$. Can you make these dots bigger - they are very difficult to see These equations form a $4^{\text {th }}$ order system of ordinary differential equations (ODE) and are readily solved numerically using suitable software, i.e. the MATLAB suite of ODE solvers. This model will be compared to droplet oscillation data. The $F_{d}$ term used is as given in [4]. 


\section{Methods}

\subsection{Experimental Equipment}

The laboratory apparatus and microscopic cells are shown in previous work [6]. Clean, dry compressed air was used to aerosolise distilled $\mathrm{H}_{2} \mathrm{O}$ from a 'Collison' type nebuliser. The flow of air was varied throughout the experiments to generate a range aerosol loading rates through the cell, as required to observe specific droplet behaviour on the fibre. The flow rate through the cell was continuously monitored and controlled using a flow meter (Cole Palmer, USA). The undiluted aerosol stream generated by the nebuliser had a particle concentration of $5.5 \times 10^{5} \mathrm{~cm}^{-3}$ at a mean size of $2.8 \pm 0.8 \mu \mathrm{m}$. The aerosol stream was diluted as required by an additional air stream.

The aerosol stream was fed through the cell being studied. There were two different cells used in the experiments. One, identical to that shown in the previous work [4] was used for all experiments except the experiments requiring measurement of the droplet oscillation. A second cell of the same dimensions was designed so that the droplet oscillation could be properly observed, in a plane perpendicular to the fibre. This was achieved by micro-drilling of the optical glass faces of the previous cell type, so that a fibre could be mounted between the glass faces. Diagrams of the two cells and cell configurations are given in [6].

Images of the process were recorded using a Basler high speed CCD camera (Germany) connected to a Zeiss Standard 25 light polarising microscope with a x10 objective lens. 


\subsection{Fibres and cell configurations}

All fibres used in the experiments were $7 \pm 0.5 \mu \mathrm{m}$ diameter glass. Fibre diameters were verified before placement in the cells, by microscopy (Zeiss Standard 25 light polarising microscope, Germany). All fibres were mounted vertically in the cells. There were two cell configurations [6]. [Configuration (1) enabled observation of the fibre in a plane tilted $15^{\circ}$ rearward from a plane perpendicular to the fibre. In this case, the microscope was tilted by $15^{\circ}$ in the opposing direction to ensure that the fibre remained vertical. Both ends of the fibre were mounted in the optical glass which formed the sides of the cell. Configuration (2) used a cell of similar dimensions, however the fibre was rotated $90^{\circ}$ in the cell (refer Figure 3), with the cell and the microscope orientation changed so the fibre remained vertical with respect to gravity.] all this is not very clear to me

\subsection{Experimental Procedure}

Prior to the experiments, the fibres were cleaned by flushing with acetone, followed by rinsing in distilled water and then drying in an oven to ensure that they were free from contamination. A cell with newly installed clean fibres was placed in the microscope and connected to the air/aerosol supply. Images were recorded using the CCD camera and microscope objective lens at 25-80 frames per second (fps) from commencement of the airflow through the cell. As mentioned, airflow rates were periodically altered, or stopped and restarted, or aerosol flow was discontinued with only air flow remaining, in order to observe specific features of the processes occurring. 


\subsection{Image Analysis}

Visual examination of the frames was used to determine important features/events which required further analysis. These frames were then analysed in the MATLAB Image Processing Toolbox (Mathworks, USA). Droplet edge profiles were determined using the 'Sobel' edge detection method incorporated in the toolbox. Droplet centre positions were determined using the "centroid" feature to examine droplet oscillation. Cross sectional areas of droplets were determined by calculating the number of pixels constituting the droplet image and converting to a size in $\mu \mathrm{m}^{2}$ using the known pixel size (1pixel $\left.=0.7 \mu \mathrm{m} \times 0.7 \mu \mathrm{m}\right)$. The droplets were assumed to be spherical, although this is not exactly the case, this approximation is sufficiently accurate and has been used previously [8].

\subsection{Data Analysis and Modelling}

To fully analyse the experimental oscillation data it was necessary to both filter the data and apply a Fourier transform. These techniques were used to determine the fundamental oscillation frequencies and patterns present in the data. A high pass filter was applied to the oscillation data to remove the peaks and smooth the data. The aim of this smoothing was to determine the existence of a fundamental oscillation within the data. It was found that the most effective high pass filter was of the form,

$Y_{s}^{f}=\frac{1}{3} Y_{s-1}^{o}+\frac{1}{3} Y_{s}^{o}+\frac{1}{3} Y_{s+1}^{o}$, 
where $Y_{s}^{f}$ is the filtered $Y_{s}^{o}$ value, and the -1 and +1 suffixes denote preceding and succeeding values in the data set respectively.

To perform the Fourier transform, on the experimental data, the MATLAB fft (fast fourier transform) function was used. The fft function determines the oscillation frequencies present in the data, then provides a plot of power vs. frequency - the power being the significance of each frequency in the data. Since the Fourier transform gives only frequency information, and not the amplitude or lag, it was necessary to use the frequencies obtained as an input to a curve fitting function, to obtain more complete information about the oscillation in the experimental data. The MATLAB fminsearch function was used to fit curves to the experimental data, using the frequency from the fft function as an input. The fminsearch function optimises a function fit so as to minimise the sum of squares given an initial starting point for the variables in the fitting function.

The oscillation activation $\left(\mathrm{O}_{\mathrm{A}}\right)$ and deactivation $\left(\mathrm{O}_{\mathrm{D}}\right)$ data (detailed later, cf. Figure 3 ) were analysed using a $t$-test to determine if statistically significant differences existed in the data.

To ensure the best possible correlation between the model and experimental data, the model was fitted using a Genetic Algorithm (GA) [9]. The GA input used a file containing the possible ranges for each model input parameter (including any known experimental error) and provided the best fit between model and experiment within these ranges.

\section{Results and Discussion}

Qualitatively, the processes that occur when barrel particles are captured on a fibre consist of: 
(1) the polydisperse liquid aerosols are initially captured on the upstream face of the fibre, almost immediately disappearing as they spread onto or "wet" the fibre;

(2) once sufficient aerosol has collected, barrel shaped droplets begin to grow at a regular spacing along the fibre (presumably created by Rayleigh instability breaking up the thin film). The droplets are initially axisymmetric (the classical barrel droplet);

(4) the droplets continue to grow by absorbing aerosol particles impinging onto the droplet surface. As the droplet grows, and drag force on the droplet increases, the droplet is pulled away from the fibre in the airflow direction, becoming axially asymmetric;

(5) eventually, the droplet will reach a critical size at which it begins to oscillate. Once a droplet commenced oscillation, the water (aerosol) flow could be stopped and the air flow velocity reduced, with the droplet continuing to oscillate until finally stopping at a lower velocity than which it started;

(6) The droplets continue to grow (by capturing aerosols or coalescencing with other droplets immediately above or below them on the fibre) and continue to oscillate, until they leave the fibre, either by being blown off or sliding down the fibre. There is also some slight random motion up and down the fibre, which can bring two droplets immediately below/above each other close enough to coalesce. The droplets were found to flow down at reasonably regular intervals, and almost never blew off the fibre (unlike clamshell droplets).

Figure 3 details the points at which oscillation commences $\left(\mathrm{O}_{\mathrm{A}}\right)$, and ceases $\left(\mathrm{O}_{\mathrm{D}}\right)$. This figure consists of a number of 'pairs' of activation/deactivation values for each droplet examined. 
The oscillation activation $\left(\mathrm{O}_{\mathrm{A}}\right)$ is clearly greater, but of similar slope to the deactivation point $\left(\mathrm{O}_{\mathrm{D}}\right)$. It should be noted that there was no measurable difference between the size of each droplet pair, so evaporation can be neglected since for these experiments the system was operating at $100 \%$ humidity. It will be noted that the $\mathrm{R}^{2}$ values are quite low $\left(\mathrm{O}_{A}=0.25, \mathrm{O}_{\mathrm{D}}\right.$ $=0.27$ ) however this can be accounted for by the difficulty of capturing the exact flow velocity where oscillation commenced or ceased. A $t$-test was used to determine if the two data sets were significantly different. The $t$-test showed an extremely high level of significance $\left(p=5.2 \times 10^{-7}\right)$ in the difference between the two data sets. This droplet oscillation is believed to be induced by the commencement of a transition of the flow regime, which is supported by the Re values. As can be observed from Figure 3, the oscillation generally only commences at Re values of 100 or greater. When Figure 3 is compared to Figure 5 in [6] (an equivalent figure for clamshell droplets), it will be noted that the slope of the linear fits to $\mathrm{O}_{A}$ and $\mathrm{O}_{\mathrm{D}}$ is slightly steeper for the latter and the droplet radii are much larger. It will also be noted that the vertical distance (Re) between the linear fits to $\mathrm{O}_{\mathrm{A}}$ and $\mathrm{O}_{\mathrm{D}}$ in both figures is approximately $60 \mathrm{Re}$. The oscillation will be further discussed later in the paper. 


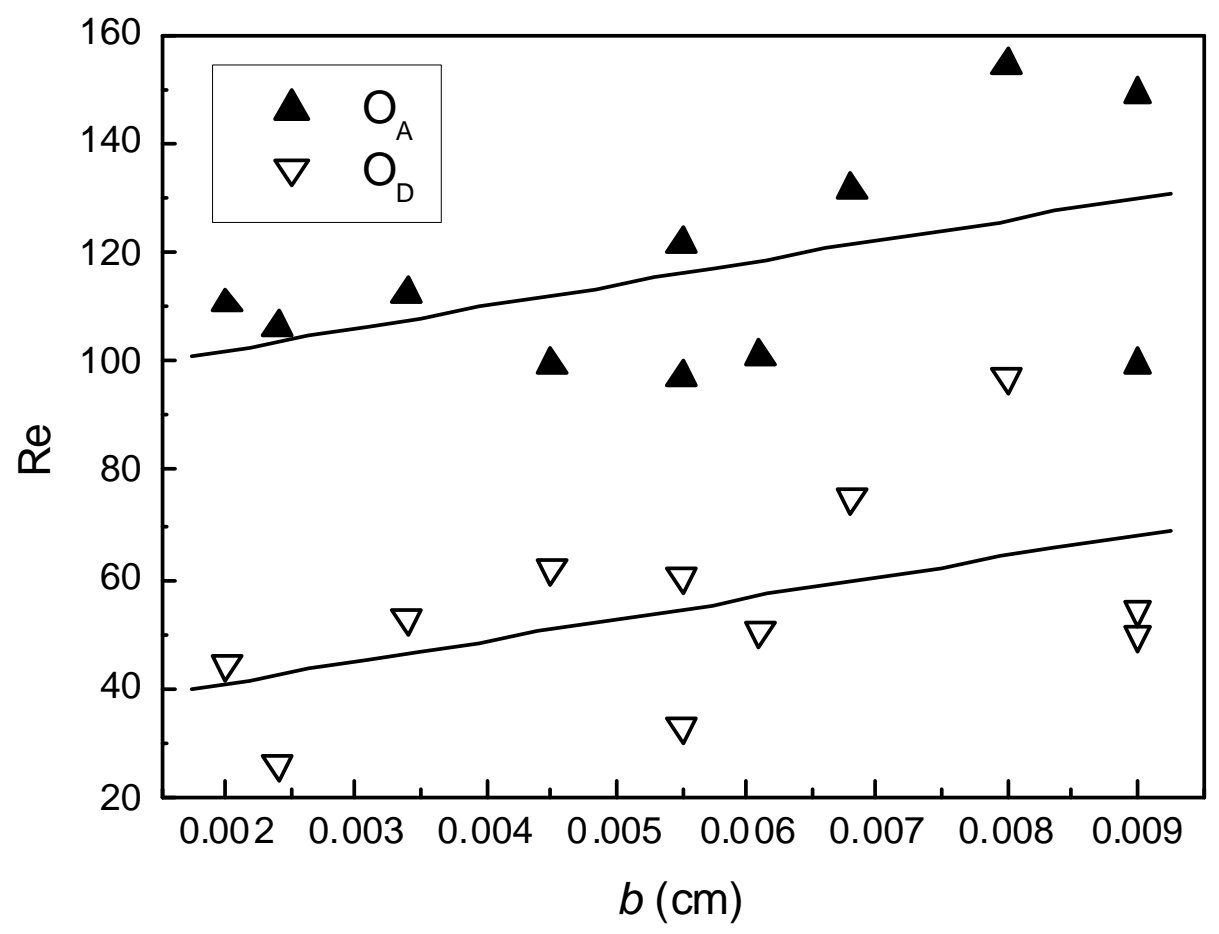

Figure 3. Activation $\left(\mathrm{O}_{\mathrm{A}}\right)$ and Deactivation $\left(\mathrm{O}_{\mathrm{D}}\right)$ of droplet oscillation as a function of Re. A straight line has been fitted to each data set using linear regression. $R^{2}\left(O_{A}\right)=0.25, R^{2}\left(O_{D}\right)$ $=0.27$. The two datasets were shown to be significantly different $\left(\mathrm{p}=5.2 \times 10^{-7}\right)$ using a $\mathrm{t}$-test $(\mathrm{t}=-7.28, \mathrm{df}=20)$.

This is a bit misleading (I think) - the figure shows the Reynolds number at which activation or deactivation occurred as a function of ?? drop size (I can't see where you have defined b)?? - so the regressions really are Reynolds numbers at which activation or deactivation occurs as functions of drop size???

Figure 4 shows the velocity at which a droplet of radius $b$ leaves the fibre. During the experiments all droplets flowed down the fibre (none were blown off), thus the droplet flows down the fibre before drag forces can break the interfacial tension holding the droplet on the fibre. The points located on the lower right hand side of the line relate to much lower air velocities, leading to much larger droplet sizes. They also correspond to a greater fibre/droplet contact area and reduced drag force allowing the droplets to flow down the fibre much more readily. Therefore all data in Figure 4 corresponds with $D_{F}$ in Figure 6 in [6]. The slope of the line fitted to Figure 4 is much steeper than the slope of the line fitted to Figure $6\left(\mathrm{D}_{\mathrm{F}}\right)$. This demonstrates both, the greatly improved drainage of barrel droplets down the fibre (due to the connecting film between droplets), 
compared to clamshell droplets, but also the fibre diameter difference again will have some effect since the barrel droplets will be unable to remain on the fibre as long.

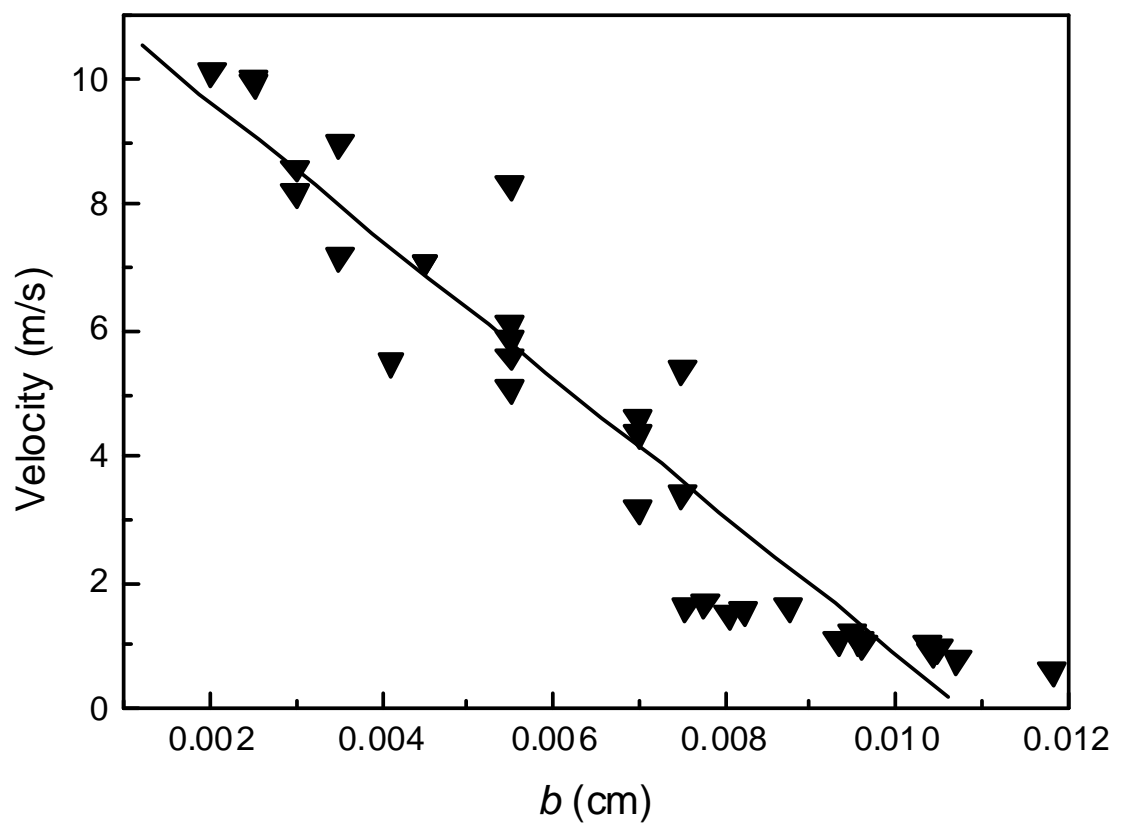

Figure 4. Droplet flow down the fibre as a function of velocity. A straight line has been fitted to each data set using linear regression. $\mathrm{R}^{2}=0.91$.

This is actually the air stream velocity at which a droplet leaves the fibre as a function of droplet size. I think you should cite the regression equation if you're going to mention the $\mathrm{R}^{2}$ - is the linear nature of the relationship telling us anything??

Another feature noted was the displacement of the mass centre of the barrel drop from its rest position as airflow velocity (and hence drag and Re) increased. Figure 5 shows the displacement of the mass centre of the droplet downstream of the fibre as a function of increasing $\operatorname{Re}$ (or equivalently air flow velocity or $\mathrm{F}_{\mathrm{D}}$ ). Measurements of the mass centre positions of 10 droplets between $b=50$ and $160 \mu \mathrm{m}$ were taken at velocities from 0 to the point at which each droplet began to oscillate, and the means of the results ( \pm one SD) are shown in Figure 5. An exponential function could be best fitted to the entire data as shown in Figure 5. The correlation is the form, 
$r=1.19 \times 10^{-7} \operatorname{Re}^{4.51}$

A linear fit has been added to all the data except the two rightmost points, which is,

$r=0.253 \operatorname{Re}-1.263$.

The linear fit was added since this portion of the graph appears near linear (justified by the $\mathrm{R}^{2}$ for the fit of 0.87 ), and $r$ is related to the restoring constant $\lambda$ in equation (3) which is in a linear form. Therefore this linear $\mathrm{r}$ relationship will allow $\lambda$ to be determined. Hmmmm this sounds a bit dodgy - either you observed the onset of oscillation or you didn't - is there any physical reason suggesting that $r$ should be a linear or quartic function of $R e$ ?? The error bars in Fig 5 suggest that your results are consistent so I would dump the linear digression unless (shudder) there IS a reason why it SHOULD be linear.

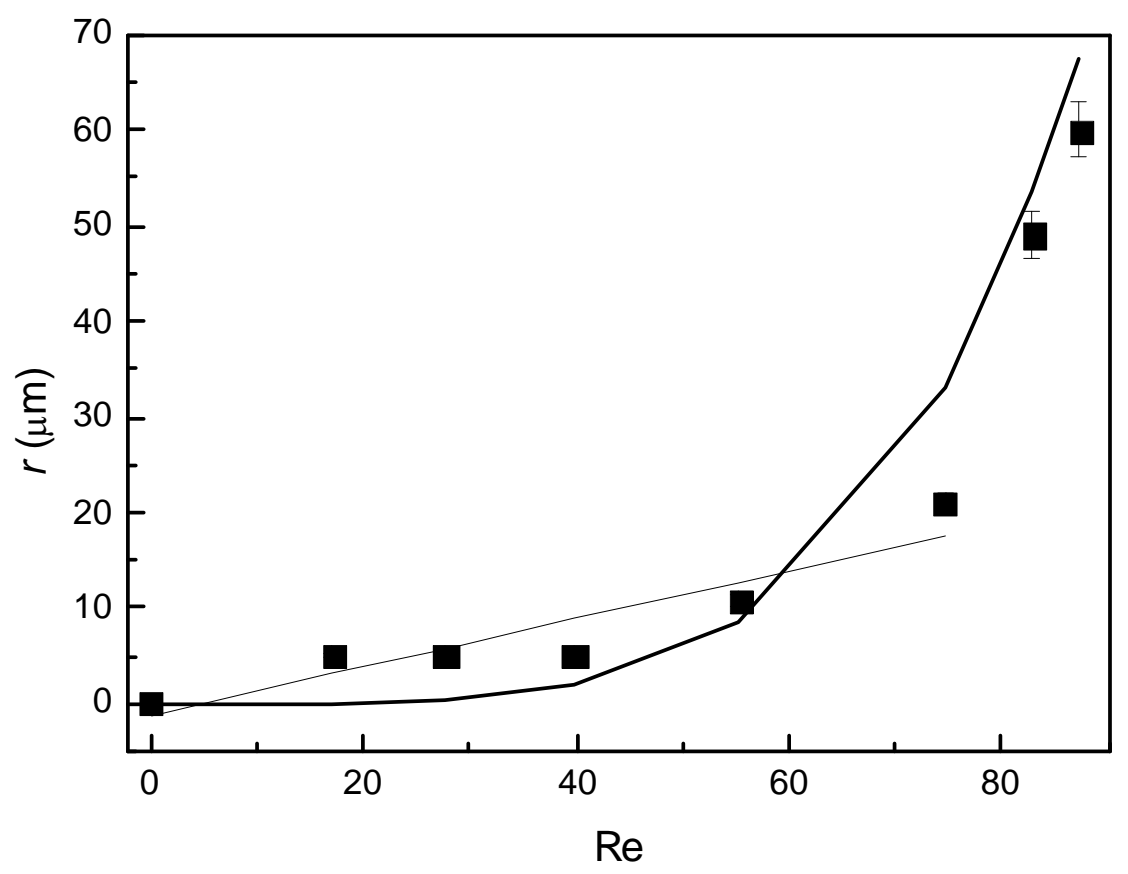


Figure 5. Barrel droplet displacement under increasing velocity prior to the commencement of oscillation. $b=50-160 \mu \mathrm{m}$. Error bars show SD of at least 10 measurements. An exponential fit has been added to the entire data (shown by the longer continuous line), and a linear fit has been added to all but the last two data points. I'd redo this with a smooth exponential curve

It will be noted that the slope of equation (7) is significantly steeper than equation (8) in [6], and the coefficient of the power term in equation (6) is greater than the power term in equation (7) in [6]. This is most likely due to the greater ability of the barrel droplet to be extended in $r$ before detachment from the fibre. Since the barrel droplet is axisymmetric at rest, it appears to allow itself to be displaced from the at-rest position more easily than an equivalent clamshell droplet, however unlike the clamshell droplet, it can be displaced almost the radius of the droplet without detachment. Therefore, clamshell droplets cannot be displaced as far without fibre detachment. The fibre contact area may have an effect, since a greater fibre/droplet contact surface will allow the clamshell droplets to have a greater resistance to displacement from the fibre.

In order to observe the droplet oscillation most accurately, a Basler high speed CCD camera was used to image the oscillation of a droplet at $80 \mathrm{fps}$, at an angle 15 degrees from a plane perpendicular to the fibre. Figures 6 and 7 show the position data for the oscillation in $r$ and $\theta_{\mathrm{o}}$ respectively of a $b=143 \mu \mathrm{m}$ (captured at $60 \mathrm{fps}$ ) 60 or $80 \mathrm{fps}$ ??? droplet in a plane perpendicular to the fibre. Note that $r$ is in $\mu \mathrm{m}$ and $\theta$ is in radians. The flow velocity around the droplet was $10.0 \mathrm{~ms}^{-1}$. The MATLAB $\mathrm{fft}$ and fminsearch functions were used to fit 
curves to the $r$ and $\theta$ data. The important (highest power) oscillation periods discovered using the fft were used as a starting point for the fminsearch fit. For the $r$ and $\theta$ data (Figures 6 and 7), it was found that there were 3-4 oscillations which had a far greater power than the others. For the highest power oscillation (lowest frequency) fit, it was found that almost identical sine functions could be fitted to either the raw or filtered data (Figures 8 and 9). However the fminsearch function proved less sensitive to the initial conditions when using filtered data.

[[[In contrast to the clamshell droplet oscillation study [6] the data contained a higher number of oscillations with an equivalent power. Therefore the oscillation is not as well defined as in the previous study. There are a number of probably reasons for this, the principal two being: (1) with the clamshell droplet on a stainless steel fibre, the fibre can be placed under slight tension in the cell, therefore making fibre oscillation with the droplet negligible - however in this case the glass fibre has a much lower tensile strength, so the oscillation of the fibre with the droplet is not insignificant; (2) since the clamshell droplet is normally located on the back of the fibre, it could be expected that its motion to be slightly more uniform, as it is unable to "pull back" into the axisymmetric position as the barrel shaped droplet can. ]]] maybe move this down one paragraph

The raw $r$ and $\theta$ data are given in Figures 6 and 7 respectively, together with the "simple" and "double" oscillations fitted to the data, denoted by the "s" and "d" subscripts respectively. The sine curves fitted to Figure 6 were,

$r_{S}=1.12 \sin (38.76 t-2.14)$,

$r_{D}=0.62 \sin (164.79 t-0.03)$, 
while the sin curves fitted to Figure 7, were of the form,

$\theta_{S}=0.36 \sin (49.71 t-2.22)$

$\theta_{D}=0.48 \sin (214.29 t-1.05)$

where $t$ is the time in seconds. The fits shown in Figures 6 and 7 are $r_{S}+r_{D}$ and $\theta_{S}+\theta_{D}$ respectively.

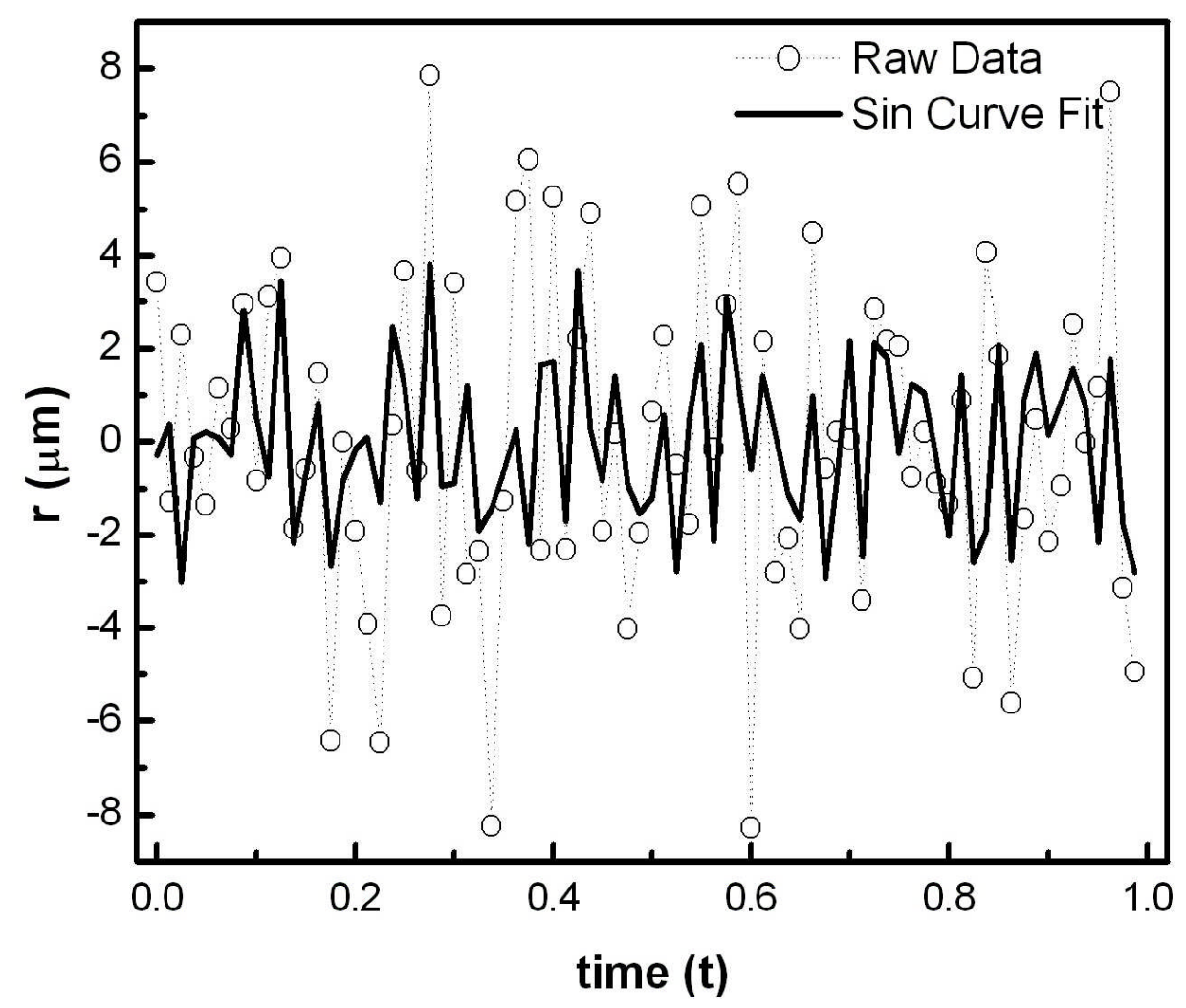

Figure 6. Oscillation of $r$ as a function of time. The raw data is shown together with a double sine curve fitted to the data. $\mathrm{R}^{2}$ between sin fit and raw data is 0.26 . 


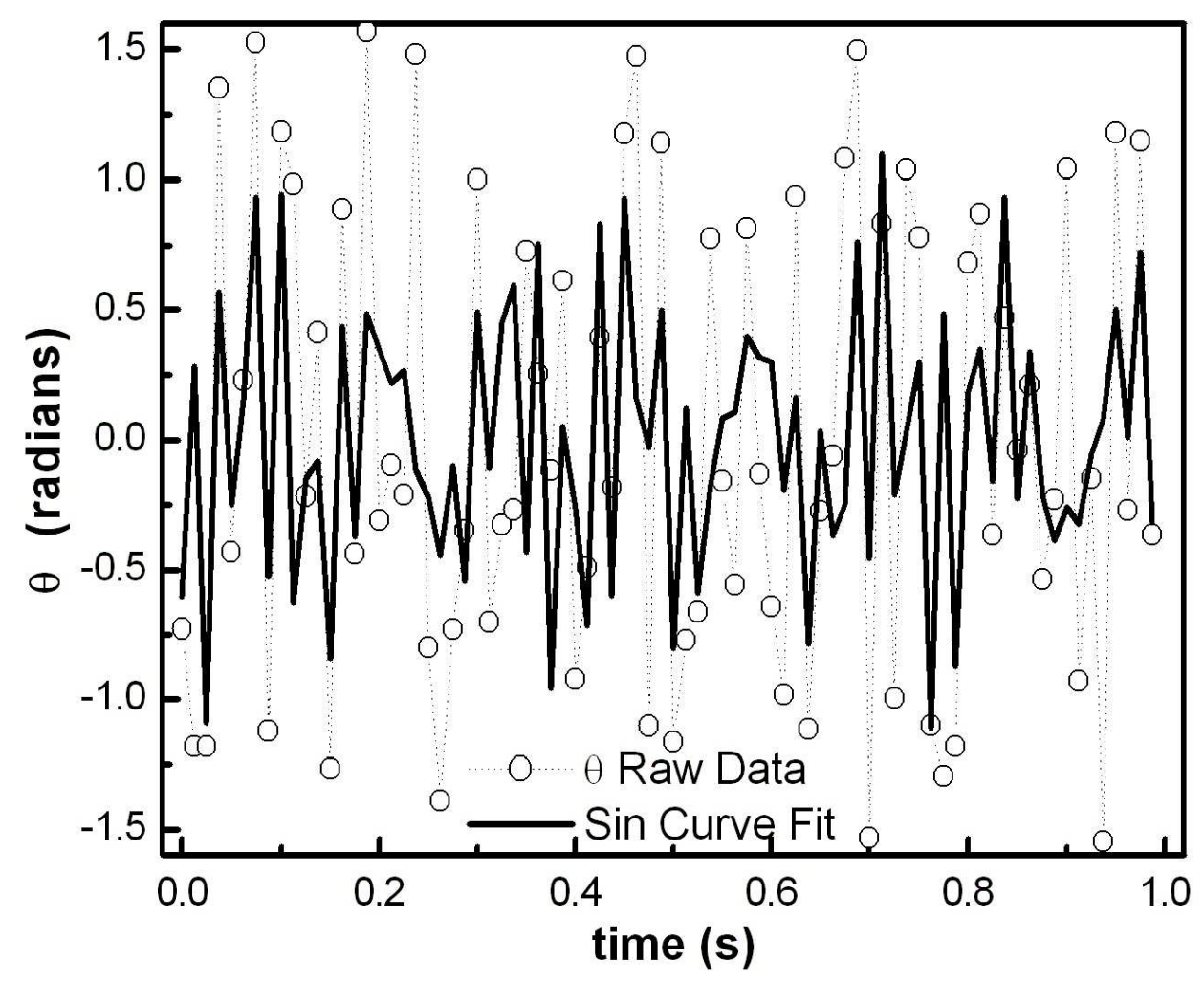

Figure 7. Oscillation of $\theta$ as a function of time. The raw data is shown together with a double sine curve fitted to the data. $\mathrm{R}^{2}$ between sin fit and raw data is 0.34 .

The simple oscillation of the droplet has a much higher frequency than in the previous study of barrel droplets [6]. However the double oscillation is of a similar frequency. This may be due to variations in droplet size (slightly smaller for this study) and flow velocity (slightly faster for this study), as well as the droplet shapes and dynamics.

Due to the more complex oscillation of the barrel droplet, the correlation coefficients (sample size corrected $-\mathrm{R}^{2}$ ) for $r$ and $\theta$ are 0.25 and 0.34 respectively, which is not ideal, but still acceptable for so complex an oscillation. Interestingly, unlike the barrel data [6], where the simple oscillations were out of phase by almost one half period, the two components of the oscillation for the barrel droplet are almost in phase with each other. 
[[[The fourier transform results for the filtered $r$ and $\theta$ data did, as mentioned, contain the "simple" oscillation (equations 9 and 10), however the high frequency "double" oscillation was removed by the filtering process, leaving a low frequency oscillation as the second highest power peak. A third oscillation was fitted, this time to the filtered data, using this second highest powers found in the fft fits for filtered $r$ and $\theta$ data.]]] I don't quite follow this - the previous fits were to raw data weren't they??? Figures 8 and 9 compare the filtered data only to the double oscillation for $r$ and $\theta$ respectively given by $r_{S}+r_{F}$ and $\theta_{S}+\theta_{F}$ respectively. The additional curves fitted to the filtered data were,

$r_{F}=0.62 \sin (19.95 t+0.13)$,

and,

$\theta_{F}=0.17 \sin (20.69 t-0.83)$. 


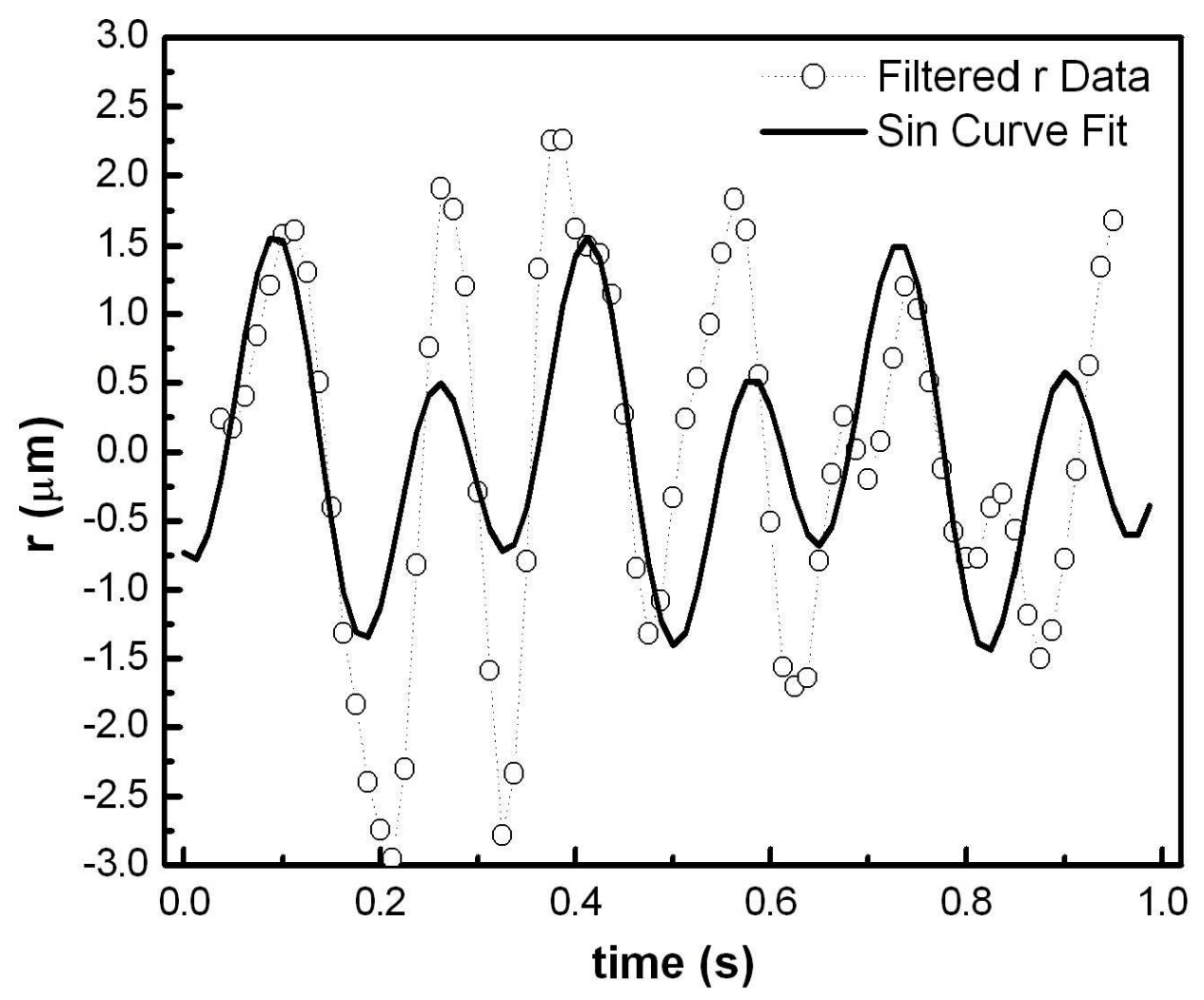

Figure 8. Filtered oscillation of $r$ as a function of time. The filtered data is shown together with a double oscillating sine curve fitted to both the major and minor oscillations. $\mathrm{R}^{2}=0.34$. 


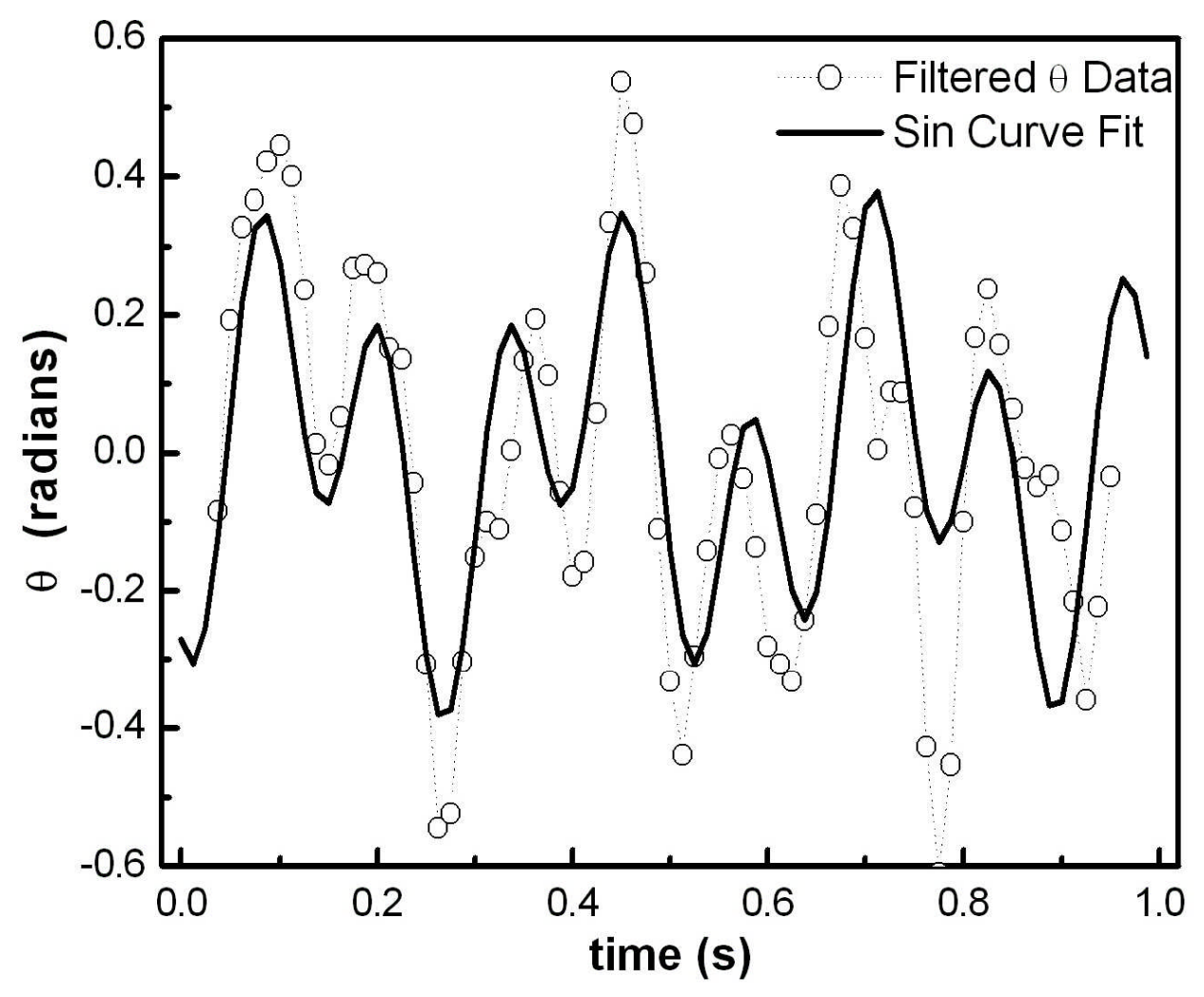

Figure 9. Filtered oscillation of $\theta$ as a function of time. The filtered data is shown together with a double oscillating sine curve fitted to both the major and minor oscillations. $\mathrm{R}^{2}=0.49$.

It will be noted that for Figures 8 and 9 the correlation between the double oscillation fit and the filtered data is improved, reinforced by $\mathrm{R}^{2}$ values of 0.34 and 0.49 for $r$ and $\theta$ respectively. It is interesting to note that the period of the filtered $r$ oscillation appears to either be changing over time, or composed of a number of other oscillations of similar frequency which were not fitted. What makes you say this?

To model the raw oscillation of $r$ and $\theta$ shown in Figures 8 and 9, initial approximations for the model input parameters $\left(m, F_{d} r, \lambda, \alpha_{o} \beta_{o}, \omega_{o}, \phi\right)$, were calculated for the droplet which was examined during the oscillation experiment. The parameters $\alpha_{o}, \beta_{o}, \omega_{o}$, and $\phi$ are, as mentioned, forcing terms representing the drag effects of the transition zone air stream. The 
value of these parameters were not known, however the amplitude, period and lag of equations (9-12) served as initial approximations. The model oscillation was fitted to the experimental oscillation data using a Genetic Algorithm (GA). The possible range of input parameters (with measurement tolerances) was used as the input bounds around which the GA would perform the fit. An estimated value of $\lambda$ was obtained from equation (8) as the droplet displacement under drag force $(r)$ pre oscillation gives a good measure of the tension force retaining the droplet on the fibre, as the displacement is a balance between the air drag on the droplet and the tension force,

As in the previous work [6], it was found during the model fitting that if the GA was executed to fit for one particular oscillation only $(r$ or $\theta)$ then it would produce a better fit, using slightly differing model input parameters. not sure what you mean here It is possible that due to the nature of the flow field, the forces acting in the $r$ and $\theta$ directions have a differing effect on or significance ???? to the nature of the respective oscillations. The best model fits to $r$ and $\theta_{\mathrm{o}}$ (when fitted individually using the GA), were obtained from the parameter sets given in Table 1 It will be noted that most of the values for each corresponding parameter in the $r$ and $\theta_{o}$ fit are very close to each other. All of the $m, F_{d}, r$, $\lambda$ values are extremely close to those obtained from experiments (and within experimental error) except for the value of $F_{d}$ which is approximately twice the experimental value. However, this may be due to the difficulty of accurately calculating drag in the transition flow region, therefore this value is acceptable. The need to increase $F_{d}$ to obtain acceptable agreement with the model was also noted in the previous barrel study [6].

Table 1 
The model output for the $r$ parameter set in Table 1 is shown in Figure 10. The model gives a reasonable fit to the filtered $r$ data $\left(\mathrm{R}^{2}\right.$ of 0.31$)$, however the fit to the raw oscillation is not as good, as expected. Unlike the previous study [6], the rapid oscillation between each time interval (camera frame rate) was not reproduced by the model, however this may be due to the faster frame rate used in this study. It will be noted that the values of the forcing terms for the $r$ fit $\left(\alpha_{\boldsymbol{o}}, \omega_{\boldsymbol{o}}\right.$ and $\left.\phi_{\boldsymbol{o}}\right)$ correspond very closely with the values of the coefficients in equation (9) (when the amplitude is converted from $\mu \mathrm{m}$ to centimetres). The model output for the $\theta$ parameter set in Table 1 is shown in Figure 11. The model gives an reasonable fit to the filtered $r$ data $\left(\mathrm{R}^{2}\right.$ of 0.23 ), and again the fit to the raw data is not as good. In both figures it is clear that the model does not capture the large amplitude oscillations, however the model explains a substantial portion of the droplet dynamics (about a quarter of the observed variation).

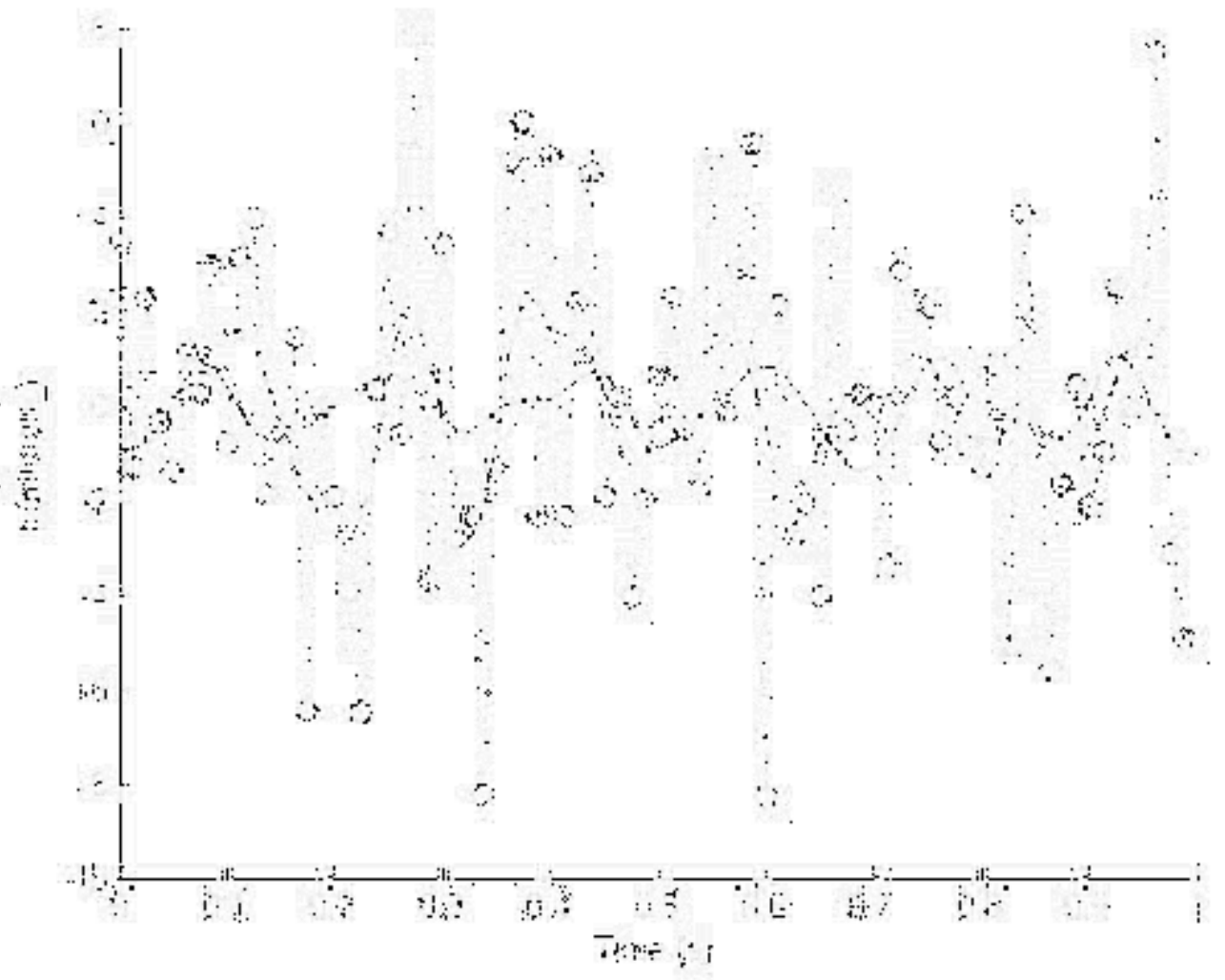


Figure 10. Model output (solid line) for oscillation of $r$ as a function of time. Raw $r$ data (dotted line with circles) and filtered data (dashed line) shown as comparison. $\mathrm{R}^{2}$ between filtered data and model 0.31 .

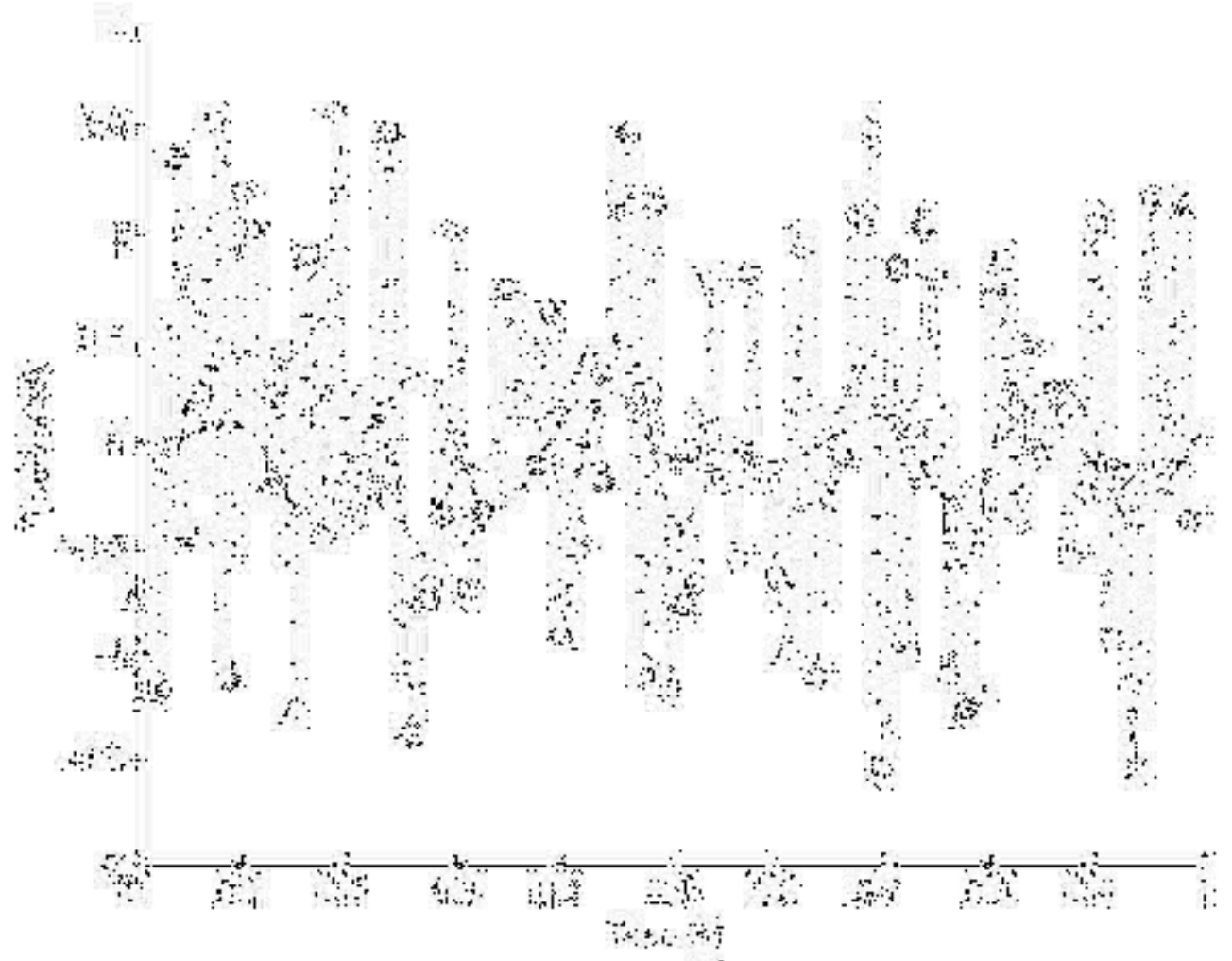

Figure 11. Model output (solid line) for oscillation of $\theta$ as a function of time. Raw $\theta$ data (dotted line with circles) and filtered data (dashed line) shown as comparison. $\mathrm{R}^{2}$ between filtered data and model 0.23. 


\section{Conclusion}

The behaviour of barrel droplets on fibres during filtration processes presents many features which are important to airflow and particle capture in filters collecting liquid aerosols. The droplet oscillation shown could greatly influence the aerosol capture characteristics inside the filter, and the air and aerosol flow paths within the filter - indeed, "blow-off" of such large droplets from filters is an important problem in mist filtration, and always occurs when the droplet is oscillating. The mathematical model which has been developed for the application, appears to be able to predict the oscillation of the droplet in the $r$ direction with reasonable accuracy (although appears better for prediction of barrel droplet oscillation) and the oscillation in the $\theta_{\mathrm{o}}$ direction with slightly reduced but acceptable accuracy, providing a different set of forcing terms are used in the input parameters. This difference in the nature of the oscillation in both directions suggests some features of the transition region flow which have a differential effect on the radial and transverse flow forces.

I think the conclusions need a bit more - quantity as well as discussion of what ALL of your results mean. You have observed several relationships in your work - can you associate them with physical processes, or importance they may have for filter design or operation???

All in all a good read and well worth the extra effort.

Roger B and Igor will comment separately. Igor was interested to know what journal you were planning to submit it to.

Cheers for now 


\section{Roger C}

I think I must have hit the format bibliography somehow - I didn’t redo the references!!!!

\section{References}

[1].Kumar, A. and S. Hartland, Shape of a Drop on a Vertical Fiber. Journal of Colloid and Interface Science, 124, 1988, 67-76.

[2].Kumar, A. and S. Hartland, Measurement of Contact Angles from the Shape of a Drop on a Vertical Fiber. Journal of Colloid and Interface Science, 136, 1990, 455-469.

[3].Mullins, B.J., R.D. Braddock, and I.E. Agranovski, Particle capture processes and evaporation on a microscopic scale in wet filters. Journal of Colloid and Interface Science, 279, 2004, 213-227.

[4].Mullins, B.J., et al., Effect of fiber orientation on fiber wetting processes. Journal of Colloid and Interface Science, 269, 2004, 449-458.

[5].Roe, R.J., Wetting of Fine Wires and Fibers by a Liquid-Film. Journal of Colloid and Interface Science, 50, 1975, 70-79.

[6].Mullins, B.J., et al., Observation and modelling of clamshell droplets on vertical fibres, subjected to gravitational and drag forces. Journal of Colloid and Interface Science, 284, 2005, 245-254.

[7].Fowles, G. and G. Cassidy, Analytical Mechanics. 6th ed, (Fort Worth: Saunders College Publishing, 1999). [8].Kirsch, A.A., Increase of Pressure-Drop in a Model Filter During Mist Filtration. Journal of Colloid and Interface Science, 64, 1978, 120-125.

[9].Holland, J., Adaptation in natural and artificial systems, (Ann Arbor: University of Michigan Press, 1975). 


\section{Acknowledgements}

The funding assistance of Smorgon Steel Tube Mills Pty. Ltd. in this work is gratefully acknowledged. 


\section{TABLES}

Table 1 - Input parameters used in the model. Units are in cgs and radians.

\begin{tabular}{lllllllll}
\hline Fit & $m$ & $F_{D}$ & $r$ & $\lambda$ & $\alpha_{0}$ & $\beta_{0}$ & $\omega_{0}$ & $\phi$ \\
\hline$r$ & $3.23 \times 10^{-4}$ & 0.25 & 0.0063 & 0.67 & $1.47 \times 10^{-4}$ & 0.58 & 36.98 & -1.43 \\
$\theta$ & $3.09 \times 10^{-4}$ & 0.26 & 0.0057 & 0.81 & $1.31 \times 10^{-4}$ & 0.42 & 41.95 & -1.53 \\
\hline
\end{tabular}

\section{FIGURES}

Figure 1. Barrel droplet on $7 \mu \mathrm{m}$ glassfibre fibre showing components of droplet oscillation. It will be noted that the droplet is no longer axisymmetric due to drag forces. When airflow velocity $=0$ the droplet returns to the axisymmetric position.

Figure 2. Diagram of droplet oscillation in a plane perpendicular to fibre, showing forces acting on droplet. Nomenclature will be used throughout. $\mathrm{F}_{\mathrm{D}}$ is drag force, $\mathrm{T}$ is the tension force (surface or line), $\mathrm{M}$ is the droplet mass centre. $\theta$ is the angle of the droplet with respect to its at rest location. 1 is the displacement of the mass centre of the droplet from the centre of the fibre in it's at rest location (for barrel droplets $l=0$ ). $r$ is the increase in droplet displacement due to drag or oscillatory forces. Airflow is in the same direction as the $\theta=0$ vector shown.

Figure 3. Activation $\left(\mathrm{O}_{\mathrm{A}}\right)$ and Deactivation $\left(\mathrm{O}_{\mathrm{D}}\right)$ of droplet oscillation as a function of Re. A straight line has been fitted to each data set using linear regression. $R^{2}\left(O_{A}\right)=0.25, R^{2}\left(O_{D}\right)$ $=0.27$. The two datasets were shown to be significantly different $\left(\mathrm{p}=5.2 \times 10^{-7}\right)$ using a t-test $(\mathrm{t}=-7.28, \mathrm{df}=20)$.

Figure 4. Droplet flow down the fibre as a function of velocity. A straight line has been fitted to each data set using linear regression. $R^{2}=0.91$.

Figure 5. Barrel droplet displacement under increasing velocity prior to the commencement of oscillation. $b=50-160 \mu \mathrm{m}$. Error bars show SD of at least 10 measurements. An exponential fit has been added to the entire data (shown by the longer continuous line), and a linear fit has been added to all but the last two data points.

Figure 6. Oscillation of $r$ as a function of time. The raw data is shown together with a double sine curve fitted to the data. $\mathrm{R}^{2}$ between sin fit and raw data is 0.26 .

Figure 7. Oscillation of $\theta$ as a function of time. The raw data is shown together with a double sine curve fitted to the data. $\mathrm{R}^{2}$ between sin fit and raw data is 0.34 . 
Figure 8. Filtered oscillation of $r$ as a function of time. The filtered data is shown together with a double oscillating sine curve fitted to both the major and minor oscillations. $\mathrm{R}^{2}=0.34$.

Figure 9. Filtered oscillation of $\theta$ as a function of time. The filtered data is shown together with a double oscillating sine curve fitted to both the major and minor oscillations. $\mathrm{R}^{2}=0.49$.

Figure 10. Model output (solid line) for oscillation of $r$ as a function of time. Raw $r$ data (dotted line with circles) and filtered data (dashed line) shown as comparison. $\mathrm{R}^{2}$ between filtered data and model 0.31 .

Figure 11. Model output (solid line) for oscillation of $\theta$ as a function of time. Raw $\theta$ data (dotted line with circles) and filtered data (dashed line) shown as comparison. $\mathrm{R}^{2}$ between filtered data and model 0.23. 